\title{
Hacia la creación del MOOC para el Mejoramiento en el Tránsito a la Educación Superior
}

Towards the creation of the MOOC for the Improvement in Traffic to Higher Education

Deicy Arévalo

Daniel Jaramillo

Pastor Benavidez $z^{3}$

Gustavo Ramírez ${ }^{4}$

Miguel Corchuelo ${ }^{5}$

\section{RESUMEN}

En Colombia y específicamente en el departamento del Cauca se presenta un bajo ingreso y una alta deserción de jóvenes de comunidades étnicas y rurales en la educación superior. En el marco de esta problemática, surgió el programa MenTES, cuya finalidad es promover que esta franja poblacional no solo acceda a la universidad, sino que se sostenga académicamente en ella. Sin embargo y sin desconocer lo destacable de este programa, se evidenció que había una falencia en temas de cobertura, sobre todo en zonas alejadas o de difícil acceso del departamento. Es así como en este trabajo se presenta el diseño del MOOC para el Mejoramiento en el Tránsito a la Educación Superior; una estrategia virtual con carácter abierto y masivo que tiene como objetivo formar docentes para que estos logren replicar la experiencia del programa MenTES en sus comunidades.

PALABRAS CLAVE: MOOC, MOOCMenTES, deserción estudiantil, acceso universitario.

\begin{abstract}
In Colombia and specifically in the department of Cauca, there is low income and high desertion of young people from ethnic and rural communities in higher education. Within the framework of this problem, the MenTES program emerged, whose purpose is to promote that this population band not only access the university, but that it be academically supported in it. However, without ignoring the remarkable nature of this program, it was evident that there was a lack of coverage, especially in remote or difficult areas of the department. This is how this paper presents the design of the MOOC for Improvement in Traffic to Higher Education; a virtual strategy with an open and massive character that aims to train teachers so that they

${ }^{1}$ Magister en Educación, Fisioterapeuta. Coordinadora Proyecto MOOCMenTES - Universidad del Cauca. Colombia. djarevalo@unicauca.edu.co

${ }^{2} \mathrm{PhD}$ (c) y Magister en Ingeniería Telemática, Ingeniero Electrónico Telecomunicaciones, dajaramillo@unicauca.edu.co.

${ }^{3} \mathrm{PhD}$ (c) y Magister en Educación, Ingeniero Electrónico y Telecomunicaciones. Docente de Educación Media- Ministerio de Educación Nacional, Colombia, pastor3571@ gmail.com

${ }^{4}$ PhD y Magister en Ingeniería Telemática, Ingeniero Electrónico Telecomunicaciones, gramirez@unicauca.edu.co.

${ }^{5} \mathrm{PhD}$ en Ciencias de la Educación, Especialista en Aprendizaje Autónomo. Licenciatura en Física y Electrónica .
\end{abstract}


can replicate the experience of the MenTES program in their communities.

KEYWORDS: MOOC, MOOCMenTES, student desertion, university access.

\section{INTRODUCCIÓN}

En las dos últimas décadas la solicitud de acceso y matricula a las Instituciones de Educación Superior -IES- ha crecido de manera importante. Sin embargo, esto no significa que se haya incrementado la cobertura. En Colombia el Ministerio de Educación Nacional -MEN-, expresa que la cobertura para el año 2013 fue de $45 \%$ (incluida la educación técnica del SENA) y el cálculo de la participación universitaria en pregrado sería del orden del 29\% («SNIES - Sistemas información»). A estas cifras se le debe agregar un alto índice de deserción estudiantil, esto implica no solo la pérdida para el estudiante, sino también para la universidad, dado que los espacios dejados por la deserción no pueden ser reemplazados por aquellos jóvenes que no tuvieron la oportunidad de ingresar a las IES. En el departamento del Cauca las situaciones citadas son frecuentes, tal como lo expresa Melo-Becerra et al., (2017). Esta situación motivó a un equipo de investigadores para proponer una iniciativa tendiente a minimizar esta problemática. Es así como en el 2014, nace el Programa para el Mejoramiento en el Tránsito a la Educación Superior -MenTES Cauca-, con el objetivo de promover el acceso a la educación superior y garantizar la sostenibilidad académica en la universidad a los estudiantes de educación media pertenecientes a zonas rurales, étnicas y vulnerables del Cauca («MenTES | InnovAcción Cauca", 2014).

Los logros del Programa MenTES han sido importantes, entre ellos se destaca que el $20 \%$ de quienes han participado en él han ingresado y se mantienen en la Educación Superior. Sin embargo, también se ha notado la necesidad de ampliar su cobertura a comunidades educativas que distan ampliamente de la ciudad de Popayán. Para ello, una primera estrategia fue el de realizar 5 multicohortes en igual número de municipios del Cauca. En el desarrollo de las actividades de esta nueva estrategia en cada uno de estos municipios, los gestores han identificado que es necesario buscar formas de beneficiar a estudiantes de zonas alejadas o de difícil acceso del departamento. En virtud de esto se planteó la siguiente pregunta: ¿Cómo incrementar la cobertura del Programa MenTES y potenciar sus beneficios en zonas rurales, étnicas y vulnerables del Cauca?

Con el ánimo de dar respuesta a esta inquietud no solo desde lo teórico sino también a partir de lo práctico, se planteó como una opción plausible el uso de los Cursos en Línea Masivos y Abiertos (MOOC, Massive Open Online Courses). Esto dado a la gran versatilidad que estas propuestas tecnológicas tienen para la educación del siglo XXI, en términos de acceso, calidad y cobertura, no solo para los estudiantes sino para las mismas Instituciones de Educación Superior (Arturo Amaya \& Alvarez, 2015; Chen, Barnett, \& Stephens, 2014; Pérez-Sanagustín, Maldonado, \& Morales, 2016).

Para integrar el Programa MenTES con las ventajas de los MOOC, surge el Proyecto MOOCMenTES, el cual no solo pretende atender de manera más óptima a los estudiantes de zonas rurales, étnicas y vulnerables del Cauca, sino que aporta a los docentes de educación media, estrategias pedagógicas y didácticas que posibiliten reflexionar acerca de su quehacer docente. Con base en esto, en las siguientes sesiones se presentan algunos de los trabajos relacionados con experiencias de MOOC, los cuales se constituyen en la base para la transformación del programa presencial en uno virtual: MOOC del Programa MenTES. Como cierre se incluyen algunos de los resultados y conclusiones del trabajo que en el momento se adelanta.

\section{Trabajos relacionados}

En (Jobe, 2013) se presenta una experiencia de un MOOC sobre los derechos humanos. El objetivo del curso fue crear una plataforma para la distribución de información en cualquier parte de Kenia de forma gratuita y así aumentar el conocimiento y la participación de sus habitantes. Esto llevó a que el diseño del MOOC manejara como temática central los derechos humanos, mediante el uso de cualquier dispositivo conectado a Internet disponible en la región. De igual forma en (Nordin, 2016), con la colaboración conjunta entre el Ministerio de Educación de Malasia y cuatro universidades públicas, se desarrolló un MOOC para el curso "Relación étnica". El mismo utilizó una versión modificada del marco ADDIE (análisis, diseño, desarrollo, implementación y evaluación) que 
llamaron "Marco de diseño instruccional ADDIE iterativo". Finalmente en (Kilde \& Gonzales, 2015), se presenta un MOOC conectivo para el desarrollo profesional de docentes de ciencias y matemáticas. Tuvo como principales protagonistas escuelas indígenas. La tecnología central de esta iniciativa fue los Connective Massive Open Online Course (cMOOC) y la finalidad consistió en construir comunidades de aprendizaje entre pares y en línea para aportar al desarrollo profesional entre profesores rurales.

\section{MOOC para el Mejoramiento en el Tránsito a la Educación Superior.}

La construcción del MOOC tuvo como insumo la documentación y materiales ya elaborados para el Programa MenTES. En tal sentido, para transformar las experiencias de este programa en un MOOC, se partió del modelo MOOC-Canvas (Figura 1).

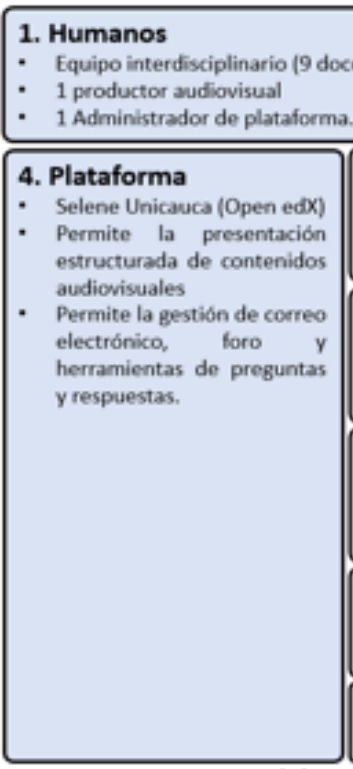

Recursos Disponibles

\begin{tabular}{|l} 
2. Intelectuales \\
- Materiales previos del programa \\
MenTES \\
Libros y Talleres
\end{tabular}

3. Equipamiento

Estudio/Laboratorio de grabación de la Universidad del Cauca

Software para la producción de contenidos (videos].

\section{Descripción general}

- Titulo: MOOC para el Mejoramiento en el Tránsito a la Educación Superior

Duración: 12 semana

Area de Educación

6. Estudiantes a los que va dirigido

- Docentes de educacion media

Docentes interesados en estrategias pedagógicas y didjicticas para brindar elementos que favorezcan el ingreso a la educación superior.

\begin{tabular}{|c|c|}
\hline $\begin{array}{l}\text { 7. Aproximaciones pedagógicas } \\
\text { - Experiencia de aprendizaje activo y } \\
\text { aprendizaje basado en problemas }\end{array}$ & $\begin{array}{l}\text { 8. Objetivos y Competencias } \\
\text { - Adquirir competencias sobre el método de formación del } \\
\text { programa MenTES. } \\
\text { - Crear estrategia pedagógicas en el aula de clase. }\end{array}$ \\
\hline $\begin{array}{l}\text { 9. Contenidos de aprendizaje } \\
\text { * } 12 \text { módulos (videos, lecturas, } \\
\text { presentaciones y foros) }\end{array}$ & $\begin{array}{l}\text { 10. Actividades de Evaluación } \\
\text { - Evaluación formativa (Test cortos) } \\
\text { - Evaluación Sumativa ( selección multiple y relación de contenidos) }\end{array}$ \\
\hline
\end{tabular}

11. Tecnologias complementarias

Youtube (contenidos en videos) y Correo electrónico

Figura 1. Diseño del MOOC utilizando el modelo MOOC-Canvas.

Este modelo está inspirado en el Business Model Canvas, el cual define once elementos interrelacionados abordados a través de preguntas, lo que ofrece un guiado visual y comprensible para los educadores durante el proceso de diseño de MOOC (Alario-Hoyos, Pérez-Sanagustín, Cormier, \& Delgado-Kloos, 2014).

En este modelo se adaptan los contenidos del programa MenTES, el cual está estructurado en función de cuatro áreas fundamentales: Habilidades comunicativas, Estrategias para el Aprendizaje, Pensamiento Lógico Matemático y Orientación Vocacional (Corchuelo et al., 2017), las que a su vez engloban en doce sesiones cuyos nombres son: Encuéntrate a ti mismo, Afina tu ritmo, Rima tus acciones, Potencia tu mente, Domina tu lengua, Aprendes cuando enseñas, Estructura tus ideas, Haz un buen negocio, Comparte noticias, Ejercítate a diario, Analiza tus decisiones y Compite sanamente. A los

\section{Decisiones de Diseño}

componentes del Programa MenTES se suma uno adicional que apoya las doce sesiones de forma transversal con las Tecnologías de Información y Comunicación TIC. Para ello ha sido importante evaluar con que infraestructura y competencias tecnológicas cuentan las comunidades educativas donde se desarrolla el Proyecto MOOCMenTES.

\section{Resultados}

Para la primera versión del MOOC MenTES se ha utilizado la plataforma de aprendizaje que brinda la Universidad del Cauca: "Selene Unicauca", la cual a su vez es una instancia de Open edX, orientada a la oferta de MOOCs. La dirección de acceso a la plataforma es: http://selene.unicauca. edu.co y el enlace a la primera versión del curso es: http://selene.unicauca.edu.co/courses/course- 
v1:MOOCMenTES+MOOC_MenTES + 2018-II/ about.

\section{Conclusiones}

Trasladar las experiencias presenciales del Programa MenTES a un MOOC, fue y sigue siendo un desafío. No es lo mismo tener un proceso de aprendizaje virtual a uno presencial y sobre todo cuando se manejan cantidades masivas de estudiantes, donde no es posible que el tutor o docente del curso tengan un contacto directo con cada estudiante, dificultando las tareas de seguimiento, realimentación e incluso la evaluación. Por tal motivo el proceso de evaluación se piensa más desde un enfoque formativo, lo vemos como una oportunidad de conocer al otro y generar conocimiento de manera colectiva. Además, es importante destacar como los MOOC se presentan como una gran alternativa para potenciar los impactos logrados en el ámbito educativo con iniciativas como el programa MenTES.

\section{AGRADECIMIENTOS}

Los autores agradecen el apoyo al proyecto VRI 4694 MOOCMenTES "Construcción de capacidades para la gestión de MOOC para la formación profesional, el desarrollo rural y nuevas generaciones de estudiantes rurales en el Mejoramiento de su Tránsito a la Educación Superior", co-financiado en el marco de alianzas rurales por el Ministerio de Educación Nacional de Colombia. Igualmente al Proyecto InnovAcción Cauca por las experiencias compartidas.

\section{REFERENCIAS}

[1]. Alario-Hoyos, C., Pérez-Sanagustín, M., Cormier, D., \& Delgado-Kloos, C. (2014). Proposal for a Conceptual Framework for Educators to Describe and Design MOOCs. Recuperado 17 de septiembre de 2018, de http://www.jucs.org/jucs_20_1/proposal_ for_a_conceptual

[2]. Arturo Amaya, A., \& Alvarez, M. V. (2015). Beneficios de los MOOC en la Educación Superior. Memorias del Encuentro Internacional de Educación a Distancia, 0 (4). Recuperado de http://www.udgvirtual.udg. $\mathrm{mx} / \mathrm{remeied} /$ index.php/memorias/article/ view/75

[3]. Chen, X., Barnett, D., \& Stephens, C. (2014). Fad or future: The advantages and challenges of massive open online courses (MOOCs). Research-to Practice Conference in Adult and Higher Education.

[4]. Corchuelo, M., Benavides, P., Arévalo, D., Ordoñez, M., León, D., Carvajal, C., \& Montenegro, L. (2017). MenTES Mejoramiento en el trásito a la educación superior en el Cauca (Vols. 1-Miguel Corchuelo). Sello Editorial Universidad del Cauca.

[5]. Jobe, W. (2013). Mobile Learning for Human Rights in Kenya: The Haki Zangu Case For Non-Formal Learning. En ICT for AntiCorruption, Democracy And Education In East Africa (pp. 67-82). Katja Sarajeva, Kista: SPIDER.

[6]. Kilde, J., \& Gonzales, L. (2015). A Connective MOOC for K-12 Science and Mathematics Teacher Professional Development in Native American Pueblo Schools. En Proceedings of the Seventh International Conference on Information and Communication Technologies and Development (pp. 46:146:4). New York, NY, USA: ACM. https:// doi.org/10.1145/2737856.2737871

[7]. MenTES | InnovAcción Cauca. (2014). Recuperado 16 de septiembre de 2018, de http://www.unicauca.edu.co/ innovaccioncauca/content/mentes

[8]. Nordin, N., Embi, M. A., \& Norman, H. (2016). Towards Envisioning the Future of Learning in Malaysia: Development of a Malaysia MOOC Based on the Iterative ADDIE Instructional Design Framework. En J. E. Luaran, J. Sardi, A. Aziz, \& N. A. Alias (Eds.), Envisioning the Future of Online Learning (pp. 269-279). Springer Singapore.

[9]. Pérez-Sanagustín, M., Maldonado, J., \& Morales, N. (2016). Estado del arte de adopción de MOOCs en la Educación Superior en América Latina y Europa. MOOC-Maker Constr. Manag. Capacit. MOOCs High. Educcation, 1. Recuperado 
de http://www.mooc-maker.org/wpcontent/files/D1.1-InformeMOOCLatamvFINALDEFINITIVO_Spanish.pdf

[10]. SNIES - Sistemas información. (s. f.). Recuperado 17 de septiembre de 2018, de https://www.mineducacion.gov. co/sistemasdeinformacion/1735/w3propertyname-2672.html 